\title{
External surfaces affected by free hydrogen in metastable austenitic stainless steels
}

\author{
Y. Katz \\ Negba, Beer Sheva, Israel
}

\begin{abstract}
Malfunction in service of contact components due to fretting fatigue enhanced mainly by the environmental interaction provided incentives to broaden and deepen the understanding of this complex time-dependent process. Metastable austenitic stainless steels affected by solid solution free hydrogen interaction, deserves further elaboration and input. In fact, the aforementioned systems combine both materials and aggressive environment that suppose to be candidates for important applications in future energy technology. As such, the whole issue of tribology turns out to be a major design topic in terms of structural integrity and service life considerations. Fretting fatigue driving force includes in addition to the remote load also the environmental interaction effects. By considering the deformation/environment interaction in metastable austenitic stainless steels at least two origins of damage are recognized, namely, sequential events connected to phase stability and hydrogen embrittlement aspects. Materials were selected from the AISI 300 group of austenitic stainless steels in general, with special attention to the relatively stable AISI 316L steel. Hydrogen was provided either by electrolytic cathodic charging or by high temperature/pressure gaseous charging. Following the current information, regarding fretting fatigue, life degradation due to internal/external hydrogen has been established. Thus, the present study is centered on tracking surface modification activated by environmental interaction confined to the near surface hydrogen affected layer. For this purpose, global and local findings were gathered in a bottom-top methodology. Standard and novel techniques were utilized including nano indentation, scratch tests and acoustic emission tracking that enabled visualization and measurements. Experimentally based, the study emphasized the local approach in contrast to the macroscopic contact mechanics approach. On a local resolution a dislocation model has been attempted, which facilitated advanced analysis as related to wear, damage evolution and near surface behavior assessment.
\end{abstract}

Keywords: austenitic stainless steel, hydrogen interaction, fretting fatigue, surface modification, localized approach. 


\section{Introduction}

Macro scale fretting fatigue in general [1] and in metastable austenitic stainless steel affected by hydrogen environment or in hydrogen pre-charged specimens in particular has been addressed by Kubota et al. [2]. The study established the destructive effects of hydrogen either on the fatigue strength or on the total service life. This unique cyclic deformation/environment interaction emphasized here the significant role of the environment, on top of the solely remote cyclic load parameter that accentuate transitions in the friction or wear behaviour $[1,3]$. Considering the role of near surface characterization affected by the environment some brief background regarding metastable austenitic stainless steel and hydrogen interaction seems to be in order. Since hydrogen/deformation interactions consist of many facets with broad implications, the wide range of intrinsic/extrinsic variables resulted in enormous number of sometimescontroversial findings and/or interpretations. Thus, the study is intentionally restricted to some relevant key-points. Regarding hydrogen/deformation interaction effects, a summary of hydrogen embrittlement (HE) will be addressed. An additional section intends to focus on small volume research activities as confined to nanoscale probing and fine features observations. In fact the current investigation is aimed to clarify the potential of such new avenues for analyzing highly localized flow and fracture events. The advances in nanomechanical characterization followed by arguments regarding its relevancy to hydrogen/deformation interactions are described. In this context nanomechanical methodology become promising in advancing tribological contact topics with attention to high resolution localized imaging information. As a background, insights as relates to HE included activities in single crystals and polycrystalline systems by utilizing global and local approaches. Nevertheless, in the current study special emphasis is paid to nano-mechanical testing engaging with tribology aspects. Clearly nanoscale contact methodology enabled additional options that can assist in cyclic evaluation supported also by nanoscratch tests information. For example, nano-indentation can extend the meaning of single point contact, providing from nano load-displacement curves, the contributions of yield excursions information to local dislocation dynamic models. Beside dislocation nucleation, the resistance of dislocations arrangement below a single contact point was explored. At this juncture the local stress field can be estimated with implications on damage evolution, failure criterion leading to transition in friction coefficient. Refinements of plastic displacement can clarify the threshold wise behaviour resulting from reversible events that were established by nanomechanical testing $[4,5]$.

\section{Material and experimental procedures}

Second phase aspects have been investigated in metastable austenitic steels while considering austenitic decomposition enhanced by hydrogen interaction. In fact, the whole AISI300 material class is unstable below the Md temperature even with no hydrogen. Moreover, hydrogen with or without a remote mechanical field can result in first order martensitic transformation. Studies in AISI 304L, 
316L and 310 stainless steels that covered a relatively wide range of phase stability have defined the important role of second phases in shaping the mechanical degradation degree [6-8]. Standard mechanical tests were applied supplemented by X-ray diffraction and Mossbauer spectroscopy analysis. Austenite decomposition was tracked with attempt to explore the transformation reaction. Only briefly, these global tests were conducted on AISI 304L, 316L and 310 martensitic stainless steels with characterization efforts as related to both, the bulk and the surface. Issues like surface relief, delayed micro cracking and local strains were all included. The nanomechanical tests were conducted mainly on the $316 \mathrm{~L}$ system. Specimen preparations involved electro chemical following mechanical polishing. Specimens were then electrochemically hydrogen charged in $1 \mathrm{MNaOH}$ for $4-6 \mathrm{~h}$ prior to testing. Charging current densities ranged from 10 to $500 \mathrm{~mA} / \mathrm{cm}^{2}$. Nanoindentation tests of the $316 \mathrm{~L}$ surfaces were conducted to a prescribed load of $1000 \mu \mathrm{N}$ by Hysitron nanoindentation instrument utilizing a $90^{\circ}$ conical indenter with $400 \mathrm{~nm}$ tip-radius of curvature. An approximate spacing of $5 \mu \mathrm{m}$ in two dimensions was maintained for adjacent indent, in microstructure consistent of grain size of 50-100 $\mu \mathrm{m}$. This allowed at least 5-10 consecutive indents to be within the same grain. For completeness, tests were performed prior to hydrogen charging, at different time stages namely, instantly after charging and one day after charging. Loaddisplacement of nanoindentation curves were supplemented by Atomic Force Microscopy (AFM) observations that enabled also measurements of indentation induced slip traces. Beside the important information of yield excursions; more was achieved by nano-scratch tests enhancing plastic deformation of finefeatures. Scratch tests were performed with the Micro-Mechanical Tester (MMT) using a $90^{\circ}$ conical indenter with $1 \mu \mathrm{m}$ radius of the tip curvature. A typical test included the following steps. The indenter was driven into the surface with the horizontal and the vertical displacements increasing at the rates of $1 \mu \mathrm{m} / \mathrm{s}$ and $7 \mathrm{~nm} / \mathrm{s}$. With the prescribed scratch length of $200 \mu \mathrm{m}$ the resulting normal force increased from 0 to $40-50 \mathrm{mN}$, depending on the local surface inclination. The maximum penetration depth scaled up to $1.5 \mu \mathrm{m}$. With the Scanning Electron Microscopy (SEM) an overview of scratch grooves were taken at different magnifications. Based on the recorded load vs. horizontal distance, loads corresponding to selected scratch segments could be estimated. For comparison of the hydrogen effects, scratch segments corresponding to nearly normal loads were selected. Again, the AFM provided quantitative measures of localized plastic deformation in addition to early cracks formation. Measurements by AFM of localized slip became a quantitative characterization based on surface slip height and spacing that enables to assess the localized strain. This methodology can provide interpretation of interfaces stress failure, namely pile-up considerations or plastic instability via slip band or void instability.

\section{Experimental results}

Global findings indicated that 304L, 316L and 310 austenitic stainless steels, differ in the phase stability degree. Considering hydrogen interaction even with 
no thermomechanical driving force, martensitic transformation occurred. Previous activities [6-8] have adopted also quantitative programs. For example, $\mathrm{X}$-ray diffraction and Mossbauer spectroscopy were aimed to determine the austenite decomposition products resulting from thermo-mechanical driving force with and with no hydrogen interaction or even from both. As such, the decomposition phase reaction was established in addition to the near surface affected layer thickness under specific gas fugacity or specific crack-tip environment conditions.

$$
\gamma \rightarrow \varepsilon^{\prime}+\alpha^{\prime}
$$

Where $\gamma$ is the austenitic phase and $\varepsilon^{\prime}$ and $\alpha^{\prime}$ are the closed packed hexagonal and body centered martensitic phases respectively. The quantitative X-ray and Mossbauer analysis in terms of phase's volume fraction remained highly consistent and slight differences were experimentally dependent (Fig. 1 and 2).
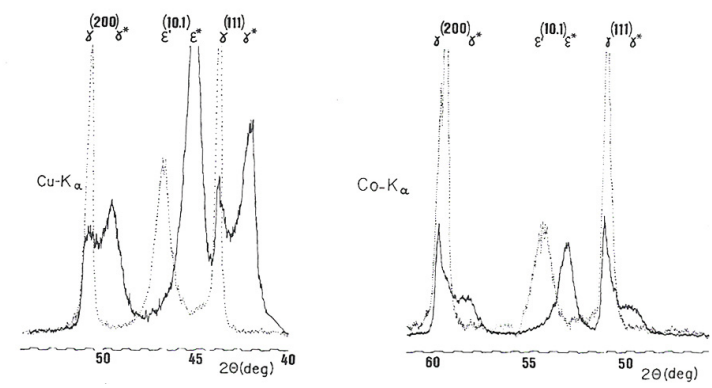

Figure 1: $\quad \mathrm{X}$-ray diffraction, 304L for different penetration power (a) charged $100 \mathrm{~min}$ at $60 \mathrm{~mA} / \mathrm{c} 2$ (b) charged $180 \mathrm{~min}$ at $100 \mathrm{~mA} / \mathrm{c} 2$.

Information regarding the near surface affected layer is highly important regarding contact wear. In addition, sequential events either instantaneous or delayed were explored. Here, events like localized plastic displacement, surface relief, microcrack nucleation and debonding were characterized. For example, in relatively low fugacity of hydrogenated AISI 304L, an affected layer of $1.14 \mu \mathrm{m}$ was established. Microscopic examinations in this material revealed pseudo expanded phases, namely $\varepsilon^{*}$ and $\gamma^{*}$ due to hydrogen uptake that transformed to $\varepsilon^{\prime}$ and $\gamma^{\prime}$ causing significant surface relief. Internal friction studies supported this behavior [9]. Finally, it became apparent that both, increased martensitic $\alpha^{\prime}$ volume fraction and multiaxial stress state accentuated the near surface degradation due to internal stress field including the critical gas release stage causing surface modification and even micro cracks nucleation beside delayed fracture. In $316 \mathrm{~L}$ the nano indentation tests resulted in reproducible displacement excursions at an average load of $200 \mu \mathrm{N}$ for non-charged samples. These were attributed to plastic initiation. Prior to the excursion load, no residual deformation exists, namely typical mechanical response that is still in the elastic regime. There was a slight difference in the average excursion load for tests 
performed in different areas of the same specimen. This was attributed to a grain orientation difference or localized pre-existing dislocations; nevertheless the average yield load was near $200 \mu \mathrm{N}$. In contrast, instantaneously after hydrogen charging the yield initiation occurred at higher values of 650 to $700 \mu \mathrm{N}$ with an elapsed time of $35 \mathrm{~min}$, the initiation load decreased to values of 300 to $350 \mu \mathrm{N}$. However, after one day, values returned back to approximately $200 \mu \mathrm{N}$ (Fig. 3).

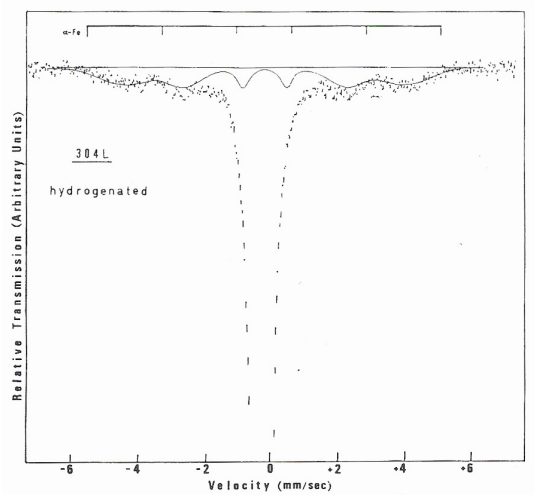

Figure 2: Mossbauer transmition spectrum of $5 \mu \mathrm{m}$ thin foil computed spectrum-solid line.
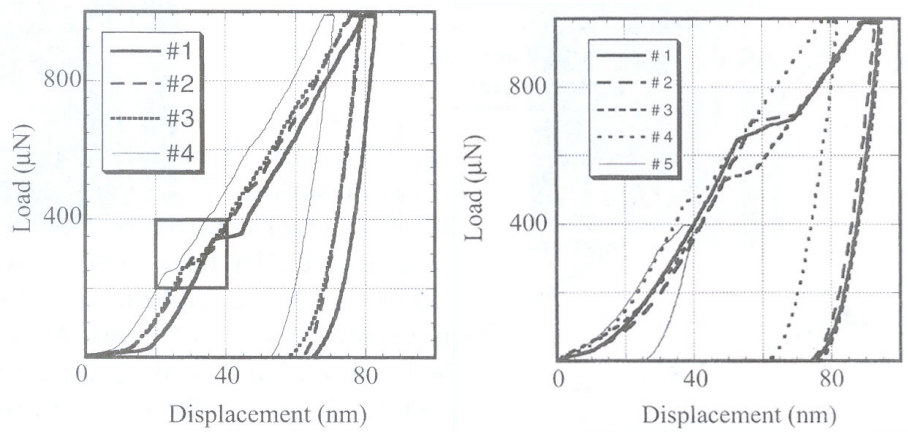

Figure 3: Nano indentation, load-displacement curve (left) no charged (right) charged. \#1-4 time increase.

Supplementing nano indentation curve analysis with AFM measures of normal indentation revealed induced slip features that were also achieved by nano scratch tests probing the hydrogen-affected surface. Here to mention that non-charged specimen were probed as well for comparative study. A comparison of quantitative measures indicated greater localization of slip with hydrogen. It is also worth noting that even with the more widely spaced slip bands, the amount of slip band upset implies a greater local plastic strain (Fig. 4). 

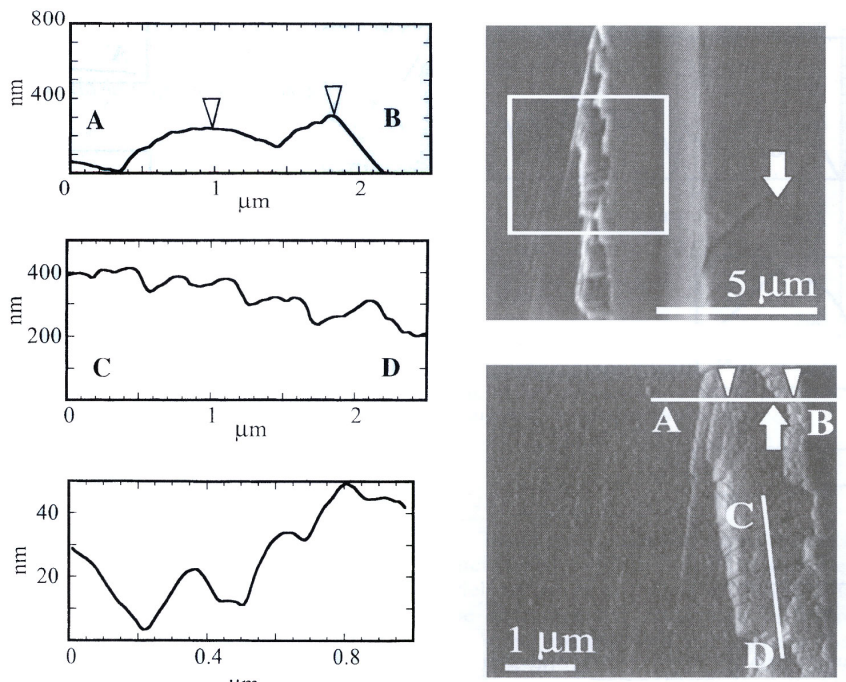

Figure 4: $\quad$ Plastic deformation features (left) AFM micrograph showing slip steps (right) SEM and AFM micrograph near nano scratch.

A determination of such surface strains has been described for fatigue induced surface slip $[10,11]$ in titanium, iron and copper. In the iron case, as cyclic work hardening occurred, it became apparent that enhanced localized plasticity may occur under hardening conditions. This is not uncommon as fatigue induced persistent slip bands in copper and $\gamma^{\prime}$ cutting in superalloy are classic examples of localization. For the scratch tracks, smaller indenter pile-up was measured in the hydrogen case. This is consistent with a more highly work hardening material compared to the non-charged case. Such effects of hydrogen on hardening in stainless steels have already been established [12]. This might lead to either decohesion along boundaries and slip planes or to plastic instability through stress or strain elevation with the enhancement of hydrogen interaction

\section{Discussion and conclusions}

Summarizing briefly the experimental findings; first, nano-indentation revealed major changes in load-displacement behavior after hydrogen charging. It seems that the initial yield increase is related to the hydrogen uptake resulting as such in stress or strain gradients. However, the time dependent stress decay is caused by the hydrogen outgassing. The hydrogen behavior can lead to misunderstanding of the exact meaning of the current reversible cycle. This apparently reversible process involves the end result of surface modification. The impact on the near surface depends on the surface condition and the hydrogen charging fugacity. Nano displacements can develop to intensive damage so important in the assessment of wear transitions of fretting behavior. Beside surface modification as mentioned already the specific contribution of hydrogen 
embrittlement aspects deserves special attention in terms of enhanced localized flow and fracture mechanisms. At this juncture it becomes apparent that nano tests methodology suggests further option into fundamental engagement as related to fretting fatigue. Nano resolution suggests connectivity to basic concepts of dislocation emission and dynamics to be translated to local stress fields. The remarkable contribution that might be achieved by nano tests as related to fundamental topics of tribology is striking. Nano-indentation representing the case of a single-point contact situation promises an experimental engagement and analysis of asperities contact wear effects. Clearly such activities open avenues for simulations assisted by dislocations based models via stress/strain field analysis. In specific materials systems, further complexities like surface morphology and statistical characteristics can be attempted in explaining contact transitions. As already been addressed by Kramer et al. [4,5], nano indentation served as powerful experimental facility exceeding more than damage description. As such, providing better understanding of the role of fatigue, micro-plasticity partition and initial methodology to include statistical aspects in damage evolution. Coming back to the deformation/environment case in metastable austenitic stainless steel systems, the hydrogen interaction affects the plastic deformation characteristics completely. This was confirmed experimentally on global and local scales. In fact, localized slip is enhanced by hydrogen uptake. In addition, martensitic transformation beside embrittlement factor involves dramatic changes at near surfaces that were defined quantitatively. Thus, implication on wear become feasible as confirmed in fretting fatigue findings. Accordingly, the following is summarized. On top of intensive activities in deformation/hydrogen embrittlement in austenitic stainless steels, including fracture mechanics methodology [13-19], nano tests have been supplemented. The extension has involved nano-indentation and micro scratch tests and other small volume effects at surfaces. Due to measured surface modification the case of fretting fatigue became highly relevant. With no surprise the findings of fretting fatigue or fretting wear tests confirmed reduction in fatigue life by hydrogen gas environment or in hydrogen pre-charged material. The potential contribution of nano tests in exploring contact wear micromechanisms could be illustrated in AFM measurements and plasticity transitions caused by hydrogen interaction. Particularly the introduction of dislocation-based models becomes possible. Hydrogen remains an aggressive environment affecting damaged sites (pitting), from identified - affected layers up to reduces fracture resistance. These sequential events at least influence strongly a fretting contact, not only in wear transition term but also in the number of cycles for fracture. In a consistent fashion the wide sense of mechanical degradation caused by hydrogen, affect also fretting fatigue behaviour. Expansion tendencies due to hydrogen transformation (in the order of $6 \%$ ) in direction parallel to the external surface are contracted by hydrogen free material under layer. Such sequential events give rise to significant internal stresses consequently the following is concluded;

(1) Metastable austenitic stainless steels interaction with hydrogen involves ample facets including the structural integrity aspects of fretting fatigue 
and contact wear degradation. This becomes as such a direct and central design concern.

(2) Searching for fundamental origins affecting contact wear, near-surface small volume probe study provided important information as related to tribological contact understanding.

(3) Particularly in AISI 316L stainless steel nano tests indicated in the load-displacement curves to explore dislocations activity particularly in comparative study including local load displacement curves.

(4) In addition, micro scratch tests revealed plastic displacements, surface perturbations, delamination and micro cracking. Such sequence of events indicated that variations in fretting fatigue with hydrogen are inevitable.

(5) Utilizing nano-tests in order to study fretting fatigue opens new and important avenues engaging not only in solely mechanical driving force but also in the role of environmental effects.

(6) In the investigated system of metastable AISI 316L stainless steel, phase stability aspects, hydrogen embrittlement combine to enhanced surface modification layer, delamination and micro-cracking.

(7) The current investigation alluded to the powerful contributions assisted by nano-tests including micro probe visualization and measurement potential in contact wear basic engagement and evaluation.

(8) The issue of threshold wise behavior needs further studies that have to be seen.

\section{Acknowledgements}

The author would like to acknowledge the collaboration of the Department of Chemical Engineering and Materials Science University of Minnesota - USA. In this framework the assistance of the Center for Interfacial Engineering is gratefully appreciated.

\section{References}

[1] Y. Kondo and M. Bodai, Study of fretting fatigue crack initiation mechanism based on local stress at contact edge, JSME, series A,63, pp 669-676,1977.

[2] M. Kubolta, N. Noyama, M. Fuerta, C. Sakae and Y. Kondo, Effect of hydrogen gas environment on fretting fatigue, J. Soc. Mat Sci. Japan, 54, pp. 1231-1236, 2005.

[3] P. Blau, Friction and wear transitions of materials, Park Ridge NJ Noyes pub.1989

[4] D.E. Krammer, H. Huang, J. Robach, J. Nelson, A. Wright, D. Bahr and W.W. Gerberich, Yield strength predictions from plastic zone around nanocontact. Acta Mater 47, pp. 333-343, 1999.

[5] D.E. Krammer, K.B. Yoder and W.W. Gerberich, Phil, Mag, 81A, p.2033, 2001. 
[6] H. Mathias, Y. Katz and S. Nadiv, Hydrogenation gas release effects in austenitic steels: Quantitative study, Melat-Hydrogen Systems, T.N. Veziroglu ed. Pergamon Press, Oxford, 225-249 1982.

[7] Y. Katz, H. Mathias and S. Nadiv, The mechanical stability of austenite in maraging steel, Metall Trans. 14A pp. 801-807 1983.

[8] Y. Katz, H, Mathias and S. Nadiv, Stability of austenitic stainless steels micro aspects transformation, Suppl. Trans. Jap, Inst. Metals. 17, pp. 3813861976.

[9] V.G. Gavriljuk, H. Hannien, A.V. Tarasenko, A.S. Tereschenko and Ullakko, Acta. Metall. Meter, 43, p.559, 1995.

[10] S. E. Harvey, P. G. Marsh and W. W. Gerberich, Acta Acta. Metall. Meter, 42, p.3493, 1994.

[11] A. Alush, A. Busiba and Y. Katz, Fatigue crack initiation stage in polycrystalline copper, ECF12, M. W. Brown, E. R. de los Rios and M. G. Miller, Eds. pp.253-258, EMA, pub, U.K, 1998.

[12] D.P.Abraham, C.J. Alstetter, Metall. Trans. 26A, p.2859, 1995.

[13] W.W. Gerberich, R.A. Oriani, M.J. Lii, T. Chen and T. Fockeke, The necessity of both plasticity and brittleness in fracture thresholds of iron. Phil. Mag. 63A, 363-76, 1991.

[14] K. Sieradzki, Theory of environmental effects on transgranular fracture, in Chemistry and Physics of Fracture. Eds. R.M. Latansiaa and R.H. Jones. Nato Asi Series E, Martinus Nijhoff Pub, Dordrecht, pp. 219-252, 1987.

[15] Y. Katz, N. Tymiak and W.W. Gerberich, Nanomechanical probes as new approaches to hydrogen/deformation interaction studies, Engin. Frac. Mecha. 68 pp. 619-646, 2001.

[16] S. H. Chen, Y. Katz and W. W. Gerberich, Crack-tip strain fields and fracture micro-plasticity in hydrogen induced cracking of Fe-3wt $\% \mathrm{Si}$ single crystals, Phil. Mag. A63 pp. 131-155, 1991.

[17] M.J. Lii, X.F. Chen, Y. Katz and W.W. Gerberich, Dislocation modelling and acoustic emission observations of alternating ductile/brittle events in Fe-3wt\% Si crystals, Acta Metall. 28, pp. 244-53, 1990.

[18] X.F. Chen, Y. Katz and W.W. Gerberich, On the directional dependency of micro-plasticity for $\{100\}$ cleavage in Fe-Si single crystals, Ser. Metall. 24, pp. $1125-30,1990$.

[19] C. W. Tien, C. J. Altstetter, CDI 92, T. Magnin, J. M. Gras, Eds, Les Editions De Physique, Les Ulis, France, 1993, p 356. 